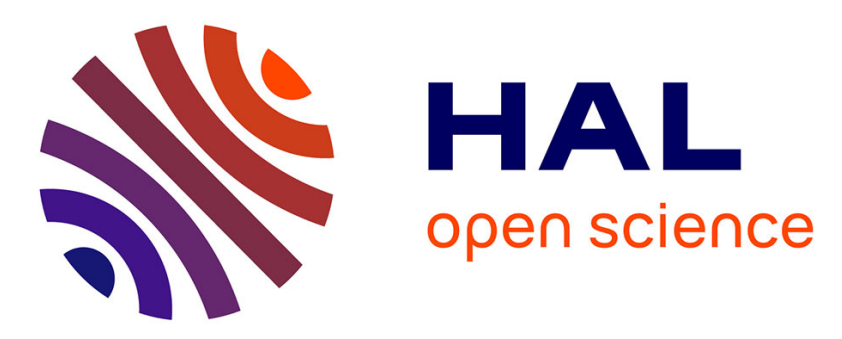

\title{
Probabilistic modeling of apparent tensors in elastostatics: A MaxEnt approach under material symmetry and stochastic boundedness constraints
}

Johann Guilleminot, Christian Soize

\section{- To cite this version:}

Johann Guilleminot, Christian Soize. Probabilistic modeling of apparent tensors in elastostatics: A MaxEnt approach under material symmetry and stochastic boundedness constraints. Probabilistic Engineering Mechanics, 2012, 28 (SI), pp.118-124. 10.1016/j.probengmech.2011.07.004 . hal-00686132

\section{HAL Id: hal-00686132 \\ https://hal.science/hal-00686132}

Submitted on 7 Apr 2012

HAL is a multi-disciplinary open access archive for the deposit and dissemination of scientific research documents, whether they are published or not. The documents may come from teaching and research institutions in France or abroad, or from public or private research centers.
L'archive ouverte pluridisciplinaire $\mathbf{H A L}$, est destinée au dépôt et à la diffusion de documents scientifiques de niveau recherche, publiés ou non, émanant des établissements d'enseignement et de recherche français ou étrangers, des laboratoires publics ou privés. 


\title{
Probabilistic modeling of apparent tensors in elastostatics: a MaxEnt approach under material symmetry and stochastic boundedness constraints
}

\author{
Johann Guilleminot*, Christian Soize \\ Université Paris-Est, Laboratoire Modélisation et Simulation Multi Echelle, MSME \\ UMR8208 CNRS, 5 Bd Descartes, 77454 Marne la Vallée, France
}

\begin{abstract}
In this work, we address the stochastic modeling of apparent elasticity tensors, for which both material symmetry and stochastic boundedness constraints have to be taken into account, in addition to the classical constraint of invertibility. We first introduce a stochastic measure of anisotropy, which is defined using metrics in the set of elasticity tensors and used for quantitatively characterizing the fulfillment of material symmetry constraints. After having define a numerical approximation for the stochastic boundedness constraint, we then propose a methodology allowing one to unify maximum entropy based models that have been previously derived by considering some of these constraints and which consists in constructing a probabilistic model for an auxiliary random variable. The latter can be interpreted as a stochastic compliance tensor, for which the available information to be used in the maximum entropy formulation can be readily deduced from the one considered for the elasticity tensor. A numerical illustration of the approach to an elastic microstructure is finally provided.
\end{abstract}

Key words: Anisotropy; Bounded tensors; Elasticity tensor; Material Symmetry; Maximum Entropy Principle; Uncertainty.

\footnotetext{
J. Guilleminot and C. Soize, Probabilistic modeling of apparent tensors in elastostatics: a MaxEnt approach under material symmetry and stochastic boundedness constraints, Probabilistic Engineering Mechanics, 28, 118-124 (2012).

${ }^{*}$ Corresponding author

Email address: johann.guilleminot@univ-paris-est.fr (Johann Guilleminot)
} 


\section{Introduction}

Stochastic multiscale modeling has become a very fast growing discipline within the past decade and as such, it gave rise to an extensive literature in both mechanics and applied mathematics, ranging from the macroscale modeling of heterogeneous materials to very first attempts of stochastic atomistic-to-continuum simulations. As a matter of fact, most of the approaches have been derived within the general scopes of uncertainty propagation and hierarchical upscaling techniques (that is, characterizing the probabilistic behavior of a complex microstructure at some relevant scale, given some description of the underlying randomness occurring at finest scales), in conjunction with the tremendous amount of past and on-going works on functional (polynomial chaos) representations for random vectorvalued quantities (see [50] [15] [45] [49]). On the other hand, it is now widely recognized that the issue of stochastic representation is also very important, in the sense that the probabilistic model associated with any random variable of interest must ensure, not only some desired properties on the solution of the stochastic boundary value problem (such as the finiteness of some of its statistical moments, for instance; see [2] and [44] for discussions in the scalar and tensor-valued cases), but also the physical consistency of the modeling procedure. Clearly, the latter issue turns out to be fundamental in any multiscale approach where information exchange across the scales is arguably crucial.

The present work deals with the probabilistic modeling of the apparent elasticity tensor for heterogeneous microstructures, defined at a so-called mesoscale (i.e. for domains whose characteristic length is smaller or of the same order as the size of the representative volume element that is usually considered in stochastic homogenization theories). From a theoretical mechanics standpoint, properties exhibited by such tensors, together with their relationship to effective ones, have been studied in the nineties by Huet and his coworkers [21] [20] (see the general review [38]). Specifically, the proposed methodology is derived within the general framework of information theory and having recourse to the Maximum Entropy (MaxEnt) principle. Such an approach, relying on random matrix theory, has been pioneered in [44] (making use of earlier derivations by the same author, obtained in the context of elastodynamics; see [42] [43]) and later followed by numerous authors (see the non-exhaustive list below). In relation with some "philosophical" issues regarding which information should reasonably be taken into account, the formal derivations thus obtained differ by the use of ad-

ditional constraints (combined to the classical constraints of normalization 
and invertibility; see section 3), integrating information related to either boundedness (see [11] and [16] for applications in the random matrix and random field cases, respectively) or material symmetry (see [46] [17]) properties for the random elasticity tensor. The aim of this study is therefore to unify these treatments and to propose a methodology allowing one to take into account all these constraints at the same time and within a nonparametric framework. For this purpose, we will have recourse to a change of variable which relaxes the boundedness constraint, thus introducing an auxiliary random variable which can be viewed as a stochastic compliance tensor and for which the probabilistic model will be finally constructed by a "translation" of the information available on the random elasticity matrix.

This paper is organized as follows. We first introduce and discuss, in section 2 , the definition of a stochastic measure of anisotropy which can be used for quantitatively characterizing, in some probabilistic sense (to be defined), the fulfillment of material symmetry constraints. Section 3 is devoted to the definition of the methodology and construction of the probabilistic model associated with the auxiliary random variable. In particular, we recall the MaxEnt principle and discuss a strategy regarding the definition of available information for this new variable. We finally provide, in section 4 , a numerical illustration of the approach.

\section{Stochastic measure of anisotropy}

\subsection{Definition}

The oldest and most widely used deterministic measure of anisotropy is based on the consideration of a scalar parameter, the definition of which depends on some given components of the elasticity tensor. Among others, the so-called Zener index, defined for crystals with cubic symmetry [51], is for instance written as $z=[C]_{44} /\left([C]_{11}-[C]_{12}\right)$, where Kelvin's notation for the elasticity tensor is assumed. While such indexes benefit from their simplicity, they suffer from a lack of universality and can hardly be extended to other situations (e.g. when the crystal exhibits weaker material symmetries or when one is interested in measuring the distance to another class than the isotropic one); see the discussions in [8] [31] [39].

Indeed, the usual anisotropy measurement can be seen as the characterization of the residual distance between a given elasticity tensor with arbitrary symmetry and its projection onto the set of isotropic elasticity tensors. Based on this observation, it follows that a more general definition of anisotropy, the latter being now understood as the distance to any material symmetry class (and not only the isotropic one), can be readily obtained 
by first defining a metric in the set $\mathbb{E} l a$ of elasticity tensors and then, by defining a projection operator onto a given subset of $\mathbb{E} l a$. A first natural distance is the Euclidean one, denoted as $\mathrm{d}_{E}$ and given for any elasticity matrices $[C]_{1}$ and $[C]_{2}$ by:

$$
\mathrm{d}_{E}\left([C]_{1},[C]_{2}\right)=\left\|[C]_{1}-[C]_{2}\right\|_{\mathrm{F}},
$$

wherein $\|\cdot\|_{\mathrm{F}}$ denotes the Frobenius norm. Alternative metrics have been derived in the literature, among which the Log-Euclidean and Riemannian ones (see [1] and [35]), denoted respectively by $\mathrm{d}_{L E}$ and $\mathrm{d}_{R}$ and defined as:

$$
\begin{gathered}
\mathrm{d}_{L E}\left([C]_{1},[C]_{2}\right)=\left\|\log [C]_{1}-\log [C]_{2}\right\|_{\mathrm{F}}, \\
\mathrm{d}_{R}\left([C]_{1},[C]_{2}\right)=\left\|\log \left([C]_{1}{ }^{-\frac{1}{2}}[C]_{2}[C]_{1}{ }^{-\frac{1}{2}}\right)\right\|_{\mathrm{F}} .
\end{gathered}
$$

Let $\mathcal{C}^{\text {sym }} \subseteq \mathbb{E} l a$ be the set of elasticity tensors with sym material symmetries (e.g. $\mathcal{C}^{\text {Iso }}$-resp. $\mathcal{C}^{\text {Trans. Iso }}$ - is the set of fourth-order isotropic -resp. transversely isotropic- elasticity tensors) and let $\mathrm{P}^{s y m}$ be the projection operator onto $\mathcal{C}^{\text {sym }}$. We then denote by $[C]^{\text {sym }}=\mathrm{P}^{\text {sym }}([C]) \in \mathcal{C}^{\text {sym }}$ the associated projection of any elasticity tensor $[C]$, defined as:

$$
[C]^{\text {sym }}=\underset{[X] \in \mathcal{C}^{\text {sym }}}{\arg \min } \mathrm{d}([C]-[X]),
$$

in which $\mathrm{d}$ is any of the metric defined above. Upon introducing a tensorial basis of $\mathcal{C}^{\text {sym }}$ (or equivalently, a parametric representation of the matrix form for an elasticity tensor in $\mathcal{C}^{\text {sym }}$ ), optimization problem (4) can be solved in a straightforward manner, yielding either closed-form expressions for the projected tensor (for the Euclidean distance) or equivalent optimization problems (formulated with respect to a finite set of parameters) when the Log-Euclidean or the Riemannian metric is used; see [36] and [5] for results expressed in matrix and vector forms, respectively. For instance, the closest isotropic approximation, in the Euclidean sense, of an arbitrary elasticity tensor $[C]$ written in Kelvin's notation is given by [14] (see also [36]):

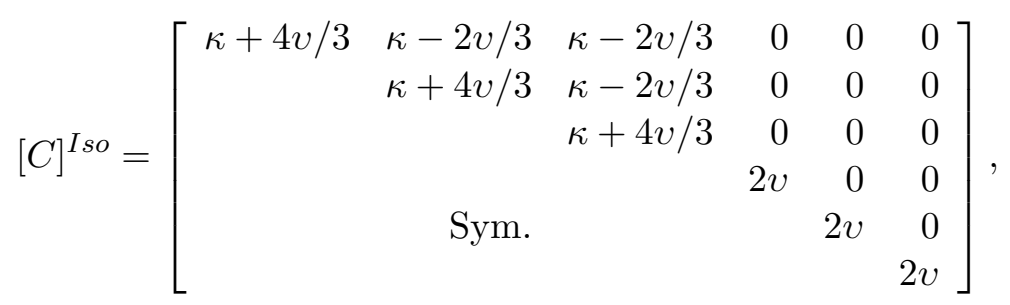


in which

$$
\kappa=\frac{1}{9}\left([C]_{11}+[C]_{22}+[C]_{33}+2\left([C]_{12}+[C]_{13}+[C]_{23}\right)\right),
$$

and

$$
\begin{aligned}
v= & \frac{1}{30}\left(2\left([C]_{11}+[C]_{22}+[C]_{33}-[C]_{12}-[C]_{23}-[C]_{31}\right)\right. \\
& \left.+3\left([C]_{44}+[C]_{55}+[C]_{66}\right)\right) .
\end{aligned}
$$

Note that this result coincides with the one derived by Fedorov, having recourse to an apparently different and more physically-sounded approach [12], and that the two different treatments were later shown to be equivalent in [37]. It is worth while to note that within a deterministic framework and for material symmetry classes involving the definition of a reference frame (e.g. for transverse isotropy, orthotropy, etc.), the computation of the closest approximation exhibiting the required symmetries (which is often referred to as the effective approximation in the literature of elasticity) necessitates an additional minimization problem, defined over all orthogonal transformation of the reference frame, to be solved; see the discussions in [6] [28] [29] [30], as well as the references therein. However, such a consideration is not relevant to this study, since the anisotropic statistical fluctuations induced by the probabilistic model can be seen as representing the randomness on both the mechanical properties and the reference frame.

Let us now consider the stochastic case, and let $[\mathbf{C}]$ be the $\mathbb{M}_{n}^{+}(\mathbb{R})$-valued random variable corresponding to the modeling of a random elasticity matrix with arbitrary symmetry. Following the previous discussions, we denote as $\left[\mathbf{C}^{\text {sym }}\right]=\mathrm{P}^{\text {sym }}\{[\mathbf{C}]\}$ the $\mathbb{M}_{n}^{+}(\mathbb{R})$-valued random variable corresponding to the projection of $[\mathbf{C}]$ onto $\mathcal{C}^{\text {sym }}$, associated with projection operator $\mathrm{P}^{\text {sym }}$ (defined with respect to any suitable metric). Consequently, a stochastic measure of anisotropy can be defined making use of the $\mathbb{R}^{+}$-valued random variable $\mu^{\text {sym }}$ defined as:

$$
\mu^{s y m}=\mathrm{d}\left([\mathbf{C}],\left[\mathbf{C}^{\text {sym }}\right]\right) .
$$

The statistical properties of $\mu^{\text {sym }}$ (and especially, its mean value) are worth characterizing and will be used in the sequel for discussing the relevance of stochastic representations for the random elasticity tensor.

\subsection{Eigensystem-based characterization of symmetries}

Having introduced the stochastic measure of anisotropy, a fundamental issue concerns the information that one may take into account in order 
some moments (e.g. the mean) of $\mu^{\text {sym }}$ to be specified. More specifically, one may answer the following question: how to characterize a given set $\mathcal{C}^{\text {sym }}$ of elasticity with sym material symmetries, so that the properties thus identified can be used in the construction of the probabilistic model? In fact, several approaches for elastic material symmetry classification and characterization have been proposed by numerous authors, among which [13] [7] [22] [47] and [10] [3] [33] to name a few.

Among the developed methodologies, the eigensystem-based characterization derived by Bóna and his coworkers [4] (see also [40] for similar considerations for transversely isotropic materials) states necessary and sufficient conditions for an elasticity tensor to belong to a given material symmetry class, in terms of properties for both the eigenvalues and the related eigenspaces. In particular, a necessary condition on the algebraic multiplicities of the eigenvalues has been derived, according to which an elasticity tensor exhibiting isotropy (resp. transverse isotropy) has one eigenvalue of multiplicity five (resp. two eigenvalues of multiplicity two) and one eigenvalue of multiplicity one (resp. two eigenvalues of multiplicity one) for instance. In order to take advantage of this property, eigenvalues are assumed to be sorted in increasing order throughout the paper (using order statistics in the stochastic case) and we define $\mathcal{S}^{s y m}$ as the set of indexes gathering the order of the eigenvalue(s) corresponding to eigenvalue(s) whose multiplicity is (are) higher than one. Let us consider, for instance, the isotropic case and assume that the eigenvalues of elasticity tensor $[C]$ are such that $\lambda_{1}=\ldots=\lambda_{5}<\lambda_{6}$. We therefore set $\mathcal{S}^{I s o}=\{1, \ldots, 5\}$ and regardless of the numerical values considered, one has $\operatorname{card}\left(\mathcal{S}^{\text {Iso }}\right)=5, \operatorname{card}\left(\mathcal{S}^{\text {Trans. Iso }}\right)=4$ and $\operatorname{card}\left(\mathcal{S}^{\text {sym }}\right) \leq n-1$ in general.

\section{Overall methodology for probabilistic modeling}

\subsection{MaxEnt procedure}

Let $\mathbb{M}_{n}^{S}(\mathbb{R})$ and $\mathbb{M}_{n}^{+}(\mathbb{R})$ be the sets of all the $(n \times n)$ real symmetric matrices and the $(n \times n)$ real symmetric positive-definite matrices $\left(\mathbb{M}_{n}^{+}(\mathbb{R}) \subset\right.$ $\left.\mathbb{M}_{n}^{S}(\mathbb{R})\right)$, respectively. Let $[C] \mapsto p_{[\mathbf{C}]}([C])$ be the probability density function (p.d.f.), from $\mathbb{M}_{n}^{+}(\mathbb{R})$ into $\mathbb{R}^{+}$, defining the probability distribution $P_{[\mathbf{C}]}=p_{[\mathbf{C}]}([C]) d[C]$ (with $d[C]=2^{n(n-1) / 4} \prod_{1 \leq i \leq j \leq n} d[C]_{i j}$; see [42]) of random matrix representation $[\mathbf{C}]$ of the elasticity tensor. The construction of the p.d.f. $p_{[\mathbf{C}]}$ can be achieved by having recourse to the MaxEnt principle, which is a very efficient approach allowing for the explicit derivation of $p_{[\mathbf{C}]}$ taking into account some constraints defining available information on 
[C] [41] [23] [24] [27] [9]. Besides the usual normalization condition for the p.d.f.,

$$
\int_{\mathbb{M}_{n}^{+}(\mathbb{R})} p_{[\mathbf{C}]}([C]) \mathrm{d}[C]=1,
$$

it is assumed that $[\mathbf{C}]$ satisfies $n_{c}$ additional constraints, each of which can be written as:

$$
\mathrm{E}\left\{\mathbf{g}^{i}([\mathbf{C}])\right\}=\mathbf{f}^{i},
$$

wherein $[\mathbf{C}] \mapsto \mathbf{g}^{i}([\mathbf{C}])$ is a given measurable mapping from $\mathbb{M}_{n}^{+}(\mathbb{R})$ into an Euclidean space $\mathbb{H}_{i}$ (whose inner product is denoted by $<\cdot, \cdot>_{\mathbb{H}_{i}}$ ) and $\mathbf{f}^{i}$ is given in the image of $\mathbf{g}^{i}$. Denoting by $\mathcal{C}_{a d}$ be the set of all the probability density functions from $\mathbb{M}_{n}^{+}(\mathbb{R})$ into $\mathbb{R}^{+}$such that all the constraints are fulfilled, the MaxEnt principle then reads:

$$
p_{[\mathbf{C}]}=\underset{p \in \mathcal{C}_{a d}}{\arg \max } \mathrm{S}(p),
$$

in which the so-called Shannon's measure of entropy $\mathrm{S}(p)$ of $p$ is defined as:

$$
\mathrm{S}(p)=-\int_{\mathbb{M}_{n}^{+}(\mathbb{R})} p([C]) \ln (p([C])) d[C],
$$

wherein $\ln$ is the Neperian logarithm. Consequently, the probability density function, estimated by using the MaxEnt principle, is the function which maximizes the uncertainties over the admissible space $\mathcal{C}_{a d}$. Upon introducing a set of $\left(n_{c}+1\right)$ Lagrange multipliers $\left\{\mathcal{L}_{i}\right\}_{i=1}^{n_{c}+1}$ (in which $\mathcal{L}_{i}$ belongs to an admissible set $\mathbb{V}_{i}$ which is a subset of $\left.\mathbb{H}_{i}\right)$ associated with the $\left(n_{c}+1\right)$ constraints and making use of the Euler-Lagrange equation applied to the optimization problem (11), it can be shown that the p.d.f. estimated by the MaxEnt principle writes:

$$
p_{[\mathbf{C}]}([C])=c_{0} \exp \left(-\sum_{i=1}^{n_{c}}<\mathcal{L}_{i}, \mathbf{g}^{i}([C])>_{\mathbb{H}_{i}}\right),
$$

in which $c_{0}=\exp \left(-\mathcal{L}_{0}\right)$ and $\mathcal{L}_{0} \in \mathbb{R}$ is the Lagrange multiplier associated with the normalization condition of the p.d.f. In practice, the Lagrange multipliers may be either numerically computed, enforcing the given constraints to hold, or used as model parameters while solving an stochastic inverse identification problem, for instance. 


\subsection{Definition of the available information}

Let us first denote by $[\underline{C}]$ the nominal (expected) value of the elasticity matrix, which could correspond to the effective (homogenized) one, for instance. Note that although such a choice could generate some modeling bias, since mesoscopic realizations do not exhibit the same level of anisotropy as the macroscopic properties, numerical analysis have shown that the level of material symmetry of the mean elasticity matrix at mesoscale tends the one of the homogenized tensor [25], so that the effective matrix may be reasonably considered as a good prior model (on which a refinement can still be performed using any optimization procedure) when no database is available at the relevant scale. In terms of stochastic modeling, it is then desirable to impose the mean value of $[\mathbf{C}]$ to be equal to $[\underline{C}]$, that is to say:

$$
\mathrm{E}\{[\mathbf{C}]\}=[\underline{C}] .
$$

Furthermore, we assume the two following properties:

(i) Let $\mathcal{I}=\left\{\mathfrak{i}_{1}, \ldots, \mathfrak{i}_{m}\right\} \subseteq[1, n]$ be an indexing set of positive integers, in which $1 \leq \mathfrak{i}_{k} \leq n$ for all $k$ in $[1, m]$. The variances of $m$ selected random eigenvalues of $[\mathbf{C}]$ are prescribed through the following equality [34]:

$$
\mathrm{E}\left\{\left(\underline{\varphi}^{\mathfrak{i}_{k} \mathrm{~T}}[\mathbf{C}] \underline{\varphi}^{\mathfrak{i}_{k}}\right)^{2}\right\}=s_{\mathfrak{i}_{k}}^{2}{\underline{\lambda_{k}}}_{\mathfrak{i}_{k}}^{2}
$$

in which $\left\{\left(\underline{\lambda}_{\mathfrak{i}_{k}}, \varphi^{\mathfrak{i}_{k}}\right)\right\}_{k=1}^{m}$ are the eigenpairs of the mean model $[\underline{C}]$ corresponding to the eigenvalues with prescribed variance and $\left\{s_{\mathfrak{i}_{k}}\right\}_{k=1}^{m}$ is a set of parameters, each of which belongs to some admissible space $\mathcal{C}_{s_{i_{k}}}^{a d} \subset \mathbb{R}_{*}^{+}$which can not be explicitly described (and basically depends on the other constraints that are considered in the MaxEnt approach). From section 2.2, it follows that some statistical properties of $\mu^{\text {sym }}$ may be calibrated by setting $\mathcal{I}=\mathcal{S}^{s y m}$ and $s_{\mathfrak{i}_{k}}=\inf \left(\mathcal{C}_{s_{i_{k}}}^{a d}\right)$, since a given material symmetry class can be partly identified from the closeness of some eigenvalues. Such a choice will be illustrated later in section 4 .

(ii) Random matrix $[\mathbf{C}]$ is almost surely (a.s.) bounded from above and below by two $\mathbb{M}_{n}^{+}(\mathbb{R})$-valued random variables, denoted by $\left[\mathbf{C}_{\ell}\right]$ and $\left[\mathbf{C}_{u}\right]$ respectively:

$$
\left[\mathbf{C}_{\ell}\right]<[\mathbf{C}]<\left[\mathbf{C}_{u}\right] \text { a.s. }
$$

The existence of the two stochastic bounds in Eq. (16) can be demonstrated, in elastostatics, having recourse to minimum complementary and potential energy principles; see the pioneering work from Huet and his coworkers [21] [20] (see also [38] and the references therein). 
Indeed, it is clear from a physical point of view that the random variables $\left[\mathbf{C}_{\ell}\right],[\mathbf{C}]$ and $\left[\mathbf{C}_{u}\right]$ are jointly distributed, the statistical dependence stemming from underlying randomness occurring at finest (mesoscopic) scales. While an associated probabilistic model could certainly be constructed, its identification would required a huge amount of data that is seldom available in the context of experimental analysis. Therefore, we propose to proceed alternately using a deterministic boundedness constraint deduced from the stochastic one. To this aim, let $\left[\widetilde{C}_{\ell}\right]$ and $\left[\widetilde{C}_{u}\right]$ be the two deterministic $\mathbb{M}_{n}^{+}(\mathbb{R})$-valued matrices defined as:

$$
\begin{aligned}
& {\left[\widetilde{C}_{\ell}\right]=\underset{[X] \in \mathcal{C}_{\ell}^{a d}}{\arg \min } \mathrm{E}\left\{\left\|[X]-\left[\mathbf{C}_{\ell}\right]\right\|^{2}\right\},} \\
& {\left[\widetilde{C}_{u}\right]=\underset{[X] \in \mathcal{C}_{u}^{a d}}{\arg \min } \mathrm{E}\left\{\left\|[X]-\left[\mathbf{C}_{u}\right]\right\|^{2}\right\},}
\end{aligned}
$$

with the admissible spaces $\mathcal{C}_{\ell}^{a d}$ and $\mathcal{C}_{u}^{a d}$ being respectively given by:

$$
\begin{aligned}
& \mathcal{C}_{\ell}^{a d}=\left\{[X] \in \mathbb{M}_{n}^{+}(\mathbb{R}),[X]<\left[\mathbf{C}_{\ell}\right] \text { a.s. }\right\}, \\
& \mathcal{C}_{u}^{a d}=\left\{[X] \in \mathbb{M}_{n}^{+}(\mathbb{R}),\left[\mathbf{C}_{u}\right]<[X] \text { a.s. }\right\} .
\end{aligned}
$$

When experimental realizations of the bounds are available (or can be computed from digitalized microstructures, for instance), the optimization problems (17) and (18) can be solved by considering usual statistical estimates for the objective function and using semidefinite algorithms that are tailored to perform on $\mathbb{M}_{6}^{+}(\mathbb{R})$ [48] (such as the YALMIP toolbox for Matlab users $[32])$; see $[16]$ for an application. Since $\left[\widetilde{C}_{\ell}\right]$ and $\left[\widetilde{C}_{u}\right]$ respectively belong to $\mathcal{C}_{\ell}^{a d}$ and $\mathcal{C}_{u}^{a d}$, it follows that $\left[\widetilde{C}_{\ell}\right]<\left[\mathbf{C}_{\ell}\right]$ and $\left[\mathbf{C}_{u}\right]<\left[\widetilde{C}_{u}\right]$ a.s. From Eq. (16), it can be deduced that:

$$
\left[\widetilde{C}_{\ell}\right]<[\mathbf{C}]<\left[\widetilde{C}_{u}\right] \text { a.s. }
$$

The constraint stated by Eq. (21) can easily be accounted for within the MaxEnt procedure by introducing the two following constraints (which are modified versions of the one considered in [42] [43]) [11]:

$$
\begin{aligned}
& \mathrm{E}\left\{\ln \left(\operatorname{det}\left([\mathbf{C}]-\left[\widetilde{C}_{\ell}\right]\right)\right)\right\}=\beta_{\ell},\left|\beta_{\ell}\right|<+\infty, \\
& \mathrm{E}\left\{\ln \left(\operatorname{det}\left(\left[\widetilde{C}_{u}\right]-[\mathbf{C}]\right)\right)\right\}=\beta_{u},\left|\beta_{u}\right|<+\infty .
\end{aligned}
$$

The constraints to be taken into account in the MaxEnt-based derivation are given by Eqs. (14), (15), (22) and (23) (together with normalization condition). Note that the deterministic boundedness constraint implies an uniform ellipticity condition on the random bilinear form arising in the weak formulation of the elasticity stochastic boundary value problem. 


\subsection{Probability distributions}

Following sections 3.1 and 3.2 , let $[\Lambda] \in \mathbb{M}_{n}^{S}(\mathbb{R}),\left\{\tau_{k} \in \mathbb{R}\right\}_{k=1}^{m},\left(1-\mathcal{L}_{\ell}\right) \in$ $\mathbb{R}$ and $\left(1-\mathcal{L}_{u}\right) \in \mathbb{R}$ be the Lagrange multipliers associated with constraints (14), (15), (22) and (23). From Eq. (13), it can be deduced that p.d.f. $[C] \mapsto p_{[\mathbf{C}]}([C])$ of random matrix $[\mathbf{C}]$ can be written as:

$$
\begin{aligned}
p_{[\mathbf{C}]}([C])= & \mathbb{I}_{\mathbb{M}_{n}^{+}(\mathbb{R})}([C]) k_{0} \operatorname{det}\left([C]-\left[\widetilde{C}_{\ell}\right]\right)^{\mathcal{L}_{\ell}-1} \operatorname{det}\left(\left[\widetilde{C}_{u}\right]-[C]\right)^{\mathcal{L}_{u}-1} \\
& \times \exp \left\{-\operatorname{tr}([\Lambda][C])-\sum_{k=1}^{m} \tau_{k}\left(\underline{\varphi}^{\mathfrak{i}_{k} \mathrm{~T}}[C] \underline{\varphi}^{\mathfrak{i}_{k}}\right)^{2}\right\},
\end{aligned}
$$

in which $k_{0}$ is a normalizing constant and $[C] \mapsto \mathbb{I}_{\mathbb{M}_{n}^{+}(\mathbb{R})}([C])$ is the indicator function of $\mathbb{M}_{n}^{+}(\mathbb{R})$. Clearly, p.d.f. (24) is non standard and its practical use gives rise to two main difficulties. The first critical issue is the construction of an associated random generator (for given values of the Lagrange multipliers), which may be a tricky task taking into account the form of the p.d.f. Indeed, algorithms based on the Markov Chain Monte Carlo (MCMC) method (such as Metropolis-Hastings algorithm [19]) may be used, making the robustness and easy-to-use aspect of the formulation somewhat questionable. The second difficulty, which is basically connected to the first one (since the mathematical expectations may be computed having recourse to usual statistical estimators), deals with the computation of the Lagrange multipliers, so that all the constraints (14), (15), (22) and (23) hold (up to a given precision). Beyond the fact that the generator may not be stable for all possible trial values of the multipliers, the resulting optimization problem must be solved on a space of very high dimension (that is, on $\mathbb{R}^{n(n+1) / 2+m+2}$ ), which may required a tremendous computational effort.

Subsequently, we propose to take into account constraint (21) through the following change of variable:

$$
[\mathbf{D}]=\left([\mathbf{C}]-\left[\widetilde{C}_{\ell}\right]\right)^{-1}-[\Delta \widetilde{C}]^{-1},
$$

in which $[\Delta \widetilde{C}]=\left[\widetilde{C}_{u}\right]-\left[\widetilde{C}_{\ell}\right],[\mathbf{D}]$ is a $\mathbb{M}_{n}^{+}(\mathbb{R})$-valued random variable, for which the probabilistic model has now to be derived. It is worth while to note that the almost sure positive-definiteness of random matrix $[\mathbf{D}]$ readily ensures that Eq. (21) is satisfied almost surely. From a mechanical standpoint, $[\mathbf{D}]$ can be interpreted as a random compliance tensor, for which symmetry constraints on $[\mathbf{C}]$ can be readily reported, provided that both $\left[\widetilde{C}_{\ell}\right]$ and $\left[\widetilde{C}_{u}\right]$ belong to $\mathcal{C}^{s y m}$. It follows that the computation of the bounds has to be performed on new admissible spaces, denoted as $\widehat{\mathcal{C}}_{\ell}^{a d}$ and $\widehat{\mathcal{C}}_{u}^{a d}$ 
respectively, and defined as:

$$
\begin{aligned}
& \widehat{\mathcal{C}}_{\ell}^{a d}=\left\{[X] \in \mathcal{C}^{s y m},[X]<\left[\mathbf{C}_{\ell}\right] \text { a.s. }\right\}, \\
& \widehat{\mathcal{C}}_{u}^{a d}=\left\{[X] \in \mathcal{C}^{s y m},\left[\mathbf{C}_{u}\right]<[X] \text { a.s. }\right\} .
\end{aligned}
$$

Note that such a choice reduces, in most practical situations, the size of the optimization problem to be solved, since $\mathcal{C}^{s y m}$ is usually spanned by a number of parameters smaller than $n(n+1) / 2$ (unless the anisotropic class is considered, but in this case, the ensemble $S E^{+}$defined in [42] [43] may be used instead).

Let $[D] \mapsto p_{[\mathbf{D}]}([D])$ be the p.d.f. of random variable $[\mathbf{D}]$, from $\mathbb{M}_{n}^{+}(\mathbb{R})$ into $\mathbb{R}^{+}$, and let us introduce, for latter convenience, the order statistics $\rho_{1} \leq \ldots \leq \rho_{n}$ of the random eigenvalues of $[\mathbf{D}]$. In order to infer about the form of $p_{[\mathbf{D}]}$ making use of the MaxEnt principle, let us consider the following constraints:

(i) The mean model of $[\mathbf{D}]$ is prescribed:

$$
\mathrm{E}\{[\mathbf{D}]\}=[\underline{D}],
$$

in which the deterministic matrix $[\underline{D}]$ mainly depends on nominal value $[\underline{C}]$ (and more slightly on the other model parameters) and is such that:

$$
\left\|\mathrm{E}\left\{\left([\mathbf{D}]+[\Delta \widetilde{C}]^{-1}\right)^{-1}+\left[\widetilde{C}_{\ell}\right]\right\}-[\underline{C}]\right\|_{\mathrm{F}} \leq \varepsilon\|[\underline{C}]\|_{\mathrm{F}},
$$

wherein $\varepsilon$ is an arbitrary small parameter (say, $10^{-6}$ ).

(ii) Random matrix $[\mathbf{D}]$ is such that:

$$
\mathrm{E}\{\ln (\operatorname{det}([\mathbf{D}]))\}=\beta,|\beta|<+\infty,
$$

(iii) Let $\mathcal{J}=\left\{\mathfrak{j}_{1}, \ldots, \mathfrak{j}_{m}\right\}$ be a set of positive integers such that $\mathfrak{j}_{k}=n+1-\mathfrak{i}_{k}$ for $k$ all in $\{1, \ldots, m\}$. The variances of some random eigenvalues of [D], selected from constraint (15), are prescribed:

$$
\mathrm{E}\left\{\left(\underline{\boldsymbol{\psi}}^{\mathrm{j}_{k} \mathrm{~T}}[\mathbf{D}] \underline{\boldsymbol{\psi}}^{\mathrm{j}_{k}}\right)^{2}\right\}=r_{\mathrm{j}_{k}}^{2}{\underline{\rho_{j}}}_{\mathrm{j}_{k}}^{2}
$$

wherein $\left\{\left(\underline{\rho}_{\mathbf{j}_{k}}, \underline{\boldsymbol{\psi}}^{\mathbf{j}_{k}}\right)\right\}_{k=1}^{m}$ are the eigenpairs of $[\underline{D}]$ associated with the constrained random eigenvalues. 
The p.d.f. $p_{[\mathbf{D}]}$ then reads:

$$
\begin{aligned}
p_{[\mathbf{D}]}([D])= & \mathbb{I}_{\mathbb{M}_{n}^{+}(\mathbb{R})}([D]) k_{0}^{*} \operatorname{det}([D])^{\mathcal{L}-1} \\
& \times \exp \left\{-\operatorname{tr}([\widetilde{\Lambda}][D])-\sum_{k=1}^{m} \widetilde{\tau}_{k}\left(\underline{\boldsymbol{\psi}}^{\mathrm{j}_{k} \mathrm{~T}}[D] \underline{\boldsymbol{\psi}}^{\mathrm{j}_{k}}\right)^{2}\right\},
\end{aligned}
$$

in which $(1-\mathcal{L}) \in \mathbb{R},[\widetilde{\Lambda}] \in \mathbb{M}_{n}^{S}(\mathbb{R})$ and $\left\{\widetilde{\tau}_{k} \in \mathbb{R}\right\}_{k=1}^{m}$ are the Lagrange multipliers associated with constraints (30), (28) and (31) respectively, and $k_{0}^{*}$ is the positive constant of normalization. It follows that $[\mathbf{D}]$ belongs to the ensemble $S E^{++}$of positive-definite symmetric random matrices introduced in [34]. For the sake of brevity, the interested reader is referred to [34] regarding algebraic results and algorithms for simulating realizations of $[\mathbf{D}]$. Moreover, it can be shown that [18]:

- Parameter $\mathcal{L} \in \mathbb{R}^{+}$controls the overall level of statistical fluctuations of random matrix $[\mathbf{D}]$.

- The normalized parameter $\widetilde{\widetilde{\tau}}_{k}=\widetilde{\tau}_{k}{\underline{\mathrm{j}_{k}}}^{2} \in \mathbb{R}^{+}$has a negligible effect on the level of statistical fluctuations of $[\mathbf{D}]$. More importantly, it allows for a reduction of the variance of the associated random eigenvalue $\rho_{\mathrm{j}_{k}}$, as $\widetilde{\widetilde{\tau}}_{k} \rightarrow+\infty$.

- The components of $[\widetilde{\Lambda}]$ (which can be shown to be a diagonal matrix) can be expressed, through an integral equation, in terms of parameters $\mathcal{L}$ and $\widetilde{\widetilde{\tau}}_{k}$ and therefore, do not appear as model parameters.

In the next section, we will illustrate that following the proposed methodology (and in particular, the definition of the bounds and indexing set $\mathcal{J}$ ), parameter $\mathcal{L}$ also controls the level of fluctuations of random elasticity tensor $[\mathbf{C}]$, and that parameters $\left\{\widetilde{\widetilde{\tau}}_{k}\right\}_{k=1}^{m}$, while associated with probabilistic model on $[\mathbf{D}]$, still allows for the mean distance of $[\mathbf{C}]$ to a given material symmetry class to be specified.

\section{Application to a two-phase elastic microstructure}

\subsection{Material description}

For illustration purposes, we consider in this final section an application of the proposed methodology to the material that has been considered in [26] (see also [25] for details). The elastic microstructure, taken from food industry, is made up of a polycrystalline phase and a polymer phase, the 
elastic mechanical properties of which have been experimentally characterized (from bending tests) and are given below:

$$
E^{(m)}=2.5 \mathrm{GPa}, \nu^{(m)}=0.3, E^{(i)}=0.0025 \mathrm{GPa}, \nu^{(i)}=0.49,
$$

in which superscript $(m)$ (resp. $(i)$ ) refers to the polycrystalline (resp. polymer) phase. For the considered (i.e. tested) sample size, the industrial material processing generates fluctuations of the volume fraction $f^{(m)}$ of phase $(m)$ (where $f^{(m)}$ typically ranges over $[0.65,0.75]$ ), and both phases are basically connected (i.e. the microstructure does not exhibit a matrix-inclusion morphology). From a macroscopic point of view, the material exhibits an isotropic elastic behavior, while numerical simulations performed on mesoscopic samples show that the latter are slightly anisotropic (this property depending on the size of the mesoscopic domain).

The nominal model $[\underline{C}]$ is assumed to be the mean value of the apparent tensors (defined with respect to kinematic uniform boundary conditions) determined in [26] (see material A, Table 2, page 3970 in [26]), namely:

$$
[\underline{C}]=\left[\begin{array}{rrrrrr}
0.9100 & 0.2560 & 0.2490 & 0 & 0 & 0.0099 \\
& 0.8680 & 0.2850 & 0.0057 & 0.0042 & 0.0014 \\
& & 1.0150 & -0.0028 & 0.0071 & 0.0170 \\
& & & 0.6240 & 0.0040 & 0.0020 \\
& \text { Sym. } & & & 0.6900 & -0.0020 \\
& & & & & 0.7100
\end{array}\right] .
$$

Such a value (which is here expressed in GPa and following Kelvin's notation) ensures the consistency with the mesoscale modeling framework, as well as with the definition of material symmetry constraints, since mesoscopic realizations are still quite close from the class of isotropic elasticity tensors. In accordance with the eigensystem-based characterization of material symmetry, it is seen that $[\underline{C}]$ has five eigenvalues that are close to one another (and would basically coincide if $[\underline{C}]$ was perfectly isotropic) and one other eigenvalue of larger magnitude, so that we set $\mathcal{I}=\{1,2,3,4,5\}$. Therefore, one has $\mathcal{J}=\{2,3,4,5,6\}$ and the variance of the first eigenvalue of $[\mathbf{D}]$ is not constrained.

For the sake of simplicity, we assume that the elastic properties of the constitutive phases are deterministic, so that the lower and upper deterministic bounds can be readily obtained as:

$$
\left[\widetilde{C}_{\ell}\right]=\left(f_{\min }\left[C^{(m)}\right]^{-1}+\left(1-f_{\min }\right)\left[C^{(i)}\right]^{-1}\right)^{-1}
$$




$$
\left[\widetilde{C}_{u}\right]=f_{\max }\left[C^{(m)}\right]+\left(1-f_{\max }\right)\left[C^{(i)}\right],
$$

in which $f_{\min }=0.65$ and $f_{\max }=0.75,\left[C^{(m)}\right]$ and $\left[C^{(i)}\right]$ being the elasticity matrices of phases $(m)$ and $(i)$ respectively. Note that for a more general application (e.g. for composites made up of more than two phases or when the mechanical behavior of each phase is locally random), a numerical procedure for solving Eqs. (17)-(18) should have been used. From Eq. (33), it can be deduced that:

$$
\left[\widetilde{C}_{\ell}\right]=\left[\begin{array}{cccccc}
0.1180 & 0.1132 & 0.1132 & 0 & 0 & 0 \\
& 0.1180 & 0.1132 & 0 & 0 & 0 \\
& & 0.1180 & 0 & 0 & 0 \\
& & & 0.0048 & 0 & 0 \\
& \text { Sym. } & & & 0.0048 & 0 \\
& & & & & 0.0048
\end{array}\right]
$$

and

$$
\left[\widetilde{C}_{u}\right]=\left[\begin{array}{cccccc}
2.5347 & 1.0920 & 1.0920 & 0 & 0 & 0 \\
& 2.5347 & 1.0920 & 0 & 0 & 0 \\
& & 2.5347 & 0 & 0 & 0 \\
& & & 1.4427 & 0 & 0 \\
& \text { Sym. } & & & 1.4427 & 0 \\
& & & & & 1.4427
\end{array}\right]
$$

in GPa. It is seen that the bounds are quite far apart from each other because of the high contrast in the mechanical properties of phases $(m)$ and (i) (note that $E^{(m)} / E^{(i)}=10^{3}$ ).

\subsection{Results}

The first step of the methodology involves, for given values of parameters $\mathcal{L}$ and $\left\{\widetilde{\widetilde{\tau}}_{k}\right\}_{k=1}^{5}$, the computation of mean value $[\underline{D}]$ making use of Eq. (29). This identification can be performed solving the following optimization problem:

$$
[\underline{D}]=\underset{[X] \in \mathbb{M}_{n}^{+}(\mathbb{R})}{\arg \min } \mathcal{F}([X]),
$$

wherein the objective function $[X] \mapsto \mathcal{F}([X])$, defined on $\mathbb{M}_{n}^{+}(\mathbb{R})$ and with values in $\mathbb{R}^{+}$, is given by:

$$
\mathcal{F}([X])=\| \mathrm{E}\left\{\left(\left[\mathbf{D}\left([X] ; \mathcal{L},\left\{\widetilde{\widetilde{\tau}}_{k}\right\}_{k=1}^{m}\right]+[\Delta \widetilde{C}]^{-1}\right)^{-1}+\left[\widetilde{C}_{\ell}\right]\right\}-[\underline{C}]\left\|_{\mathrm{F}} /\right\|[\underline{C}] \|_{\mathrm{F}}\right.
$$


in which the notation $\left[\mathbf{D}\left([X] ; \mathcal{L},\left\{\widetilde{\widetilde{\tau}}_{k}\right\}_{k=1}^{m}\right]\right.$ means that the probabilistic model for random matrix $[\mathbf{D}]$ is parametrized by mean model $[X]$ and given multipliers $\mathcal{L}$ and $\left\{\widetilde{\widetilde{\tau}}_{k}\right\}_{k=1}^{m}$. Here, the nonlinear constrained optimization problem (39) is solved using a line-search algorithm performing in the neighborhood of an initial guess $\left[X^{0}\right]$ taken as:

$$
\left[X^{0}\right]=\left([\underline{C}]-\left[\widetilde{C}_{\ell}\right]\right)^{-1}-[\Delta \widetilde{C}]^{-1},
$$

the mathematical expectation being computed from the usual statistical estimator (with 50000 independent realizations of $[\mathbf{D}]$ ). For $\mathcal{L}=60$ and setting $\widetilde{\widetilde{\tau}}_{k}=\tau=500$ for all $k \in\{1, \ldots, 5\}$, the solution is found to be:

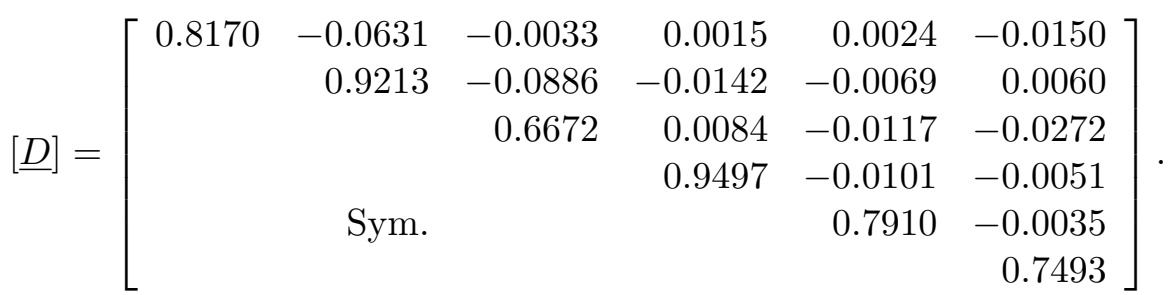

The mean value of $[\mathbf{C}]$, estimated using Eq. (25) and realizations of random compliance matrix $[\mathbf{D}]$ (generated with the parameters given above and the mean value $(42)$ ), is given by:

$$
\left[\underline{C}^{s i m}\right]=\left[\begin{array}{rrrrrr}
0.9099 & 0.2559 & 0.2489 & 0.0002 & 0.0002 & 0.0097 \\
& 0.8677 & 0.2849 & 0.0056 & 0.0043 & 0.0013 \\
& & 1.0149 & -0.0030 & 0.0072 & 0.0168 \\
& & & 0.6247 & 0.0040 & 0.0020 \\
& \text { Sym. } & & & 0.6898 & -0.0014 \\
& & & & & 0.7097
\end{array}\right] .
$$

A comparison between the computed mean value (43) and the target value (34) shows the efficiency of the approach.

Let us now briefly investigate the influence of model parameters through a parametric study. For this purpose, let $\delta_{[\mathbf{C}]}=\left(\mathrm{E}\left\{\|[\mathbf{C}]-[\underline{C}]\|_{\mathrm{F}}^{2}\right\} /\|[\underline{C}]\|_{\mathrm{F}}^{2}\right)^{1 / 2}$ be the real parameter measuring the level of statistical fluctuations of random matrix $[\mathbf{C}]$. Setting all parameters $\widetilde{\widetilde{\tau}}_{k}$ to the same value $\tau$, the plot of mapping $(\mathcal{L}, \tau) \mapsto \delta_{[\mathbf{C}]}$ is shown in Fig. 1 (note that for each selected value of $(\mathcal{L}, \tau)$, a new mean matrix $[\underline{D}]$ must be computed). It is readily seen that:

(i) For a given value of $\mathcal{L}$, parameter $\tau$ does not really affect the overall level of fluctuations of $[\mathbf{C}]$. 


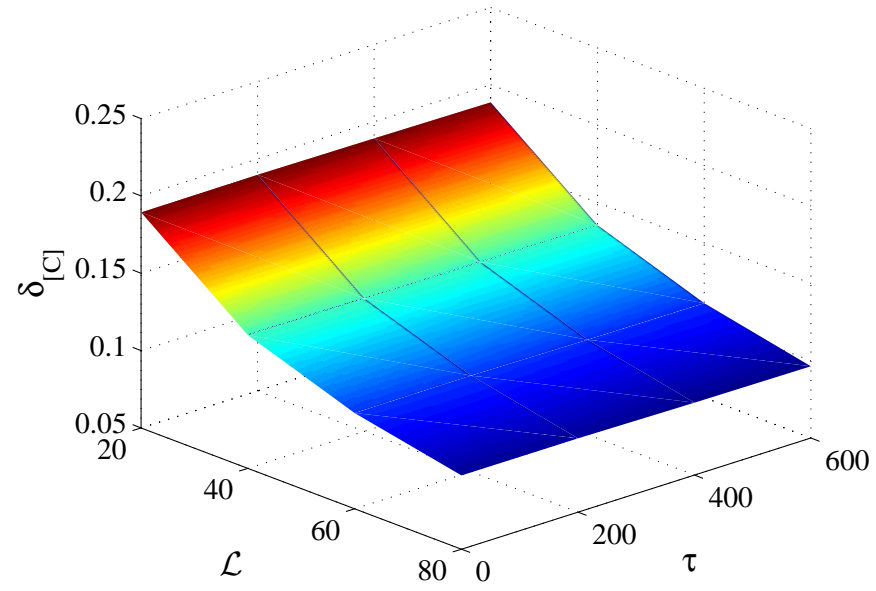

Figure 1: Plot of mapping $(\mathcal{L}, \tau) \mapsto \delta_{[\mathbf{C}]}$.

(ii) For a given value of $\tau$, decreasing the value of $\mathcal{L} \in \mathbb{R}_{*}^{+}$yields higher fluctuations, while the deterministic situation (i.e. $\delta_{[\mathbf{C}]} \rightarrow 0$ ) is recovered when $\mathcal{L} \rightarrow+\infty$.

Consequently, the value of multiplier $\mathcal{L}$ must be selected for calibrating the overall level of fluctuations of the elasticity random matrix $[\mathbf{C}]$.

In order to quantify the influence of the parametrization regarding the material symmetry constraint, let us now consider the stochastic measure of anisotropy $\mu_{E}$ defined, without loss of generality, with respect to the Euclidean distance and $\mathcal{C}^{\text {Iso }}$. The plot of mapping $(\mathcal{L}, \tau) \mapsto \mathrm{E}\left\{\mu_{E}\right\}$, representing the evolution of the mean distance to the isotropy symmetry class as a function of model parameters, is shown in Fig. 2 (note that for $\tau=0$, there is no constrained random eigenvalues and the initial theory derived in [42] [43] is recovered). It is seen that for a fixed value of $\tau$, decreasing parameter $\mathcal{L}$ yields a larger mean distance to the symmetry class, which is consistent with the previous conclusion regarding the induced variation of dispersion parameter $\delta_{[\mathbf{C}]}$ (since most of the realizations are likely to be "less isotropic"). On the other hand, for a given value of $\mathcal{L}$ (or equivalently, of $\delta_{[\mathbf{C}]}$ ), it is readily observed that increasing $\tau$ allows for a substantial reduction of the mean distance (note that further numerical experiments show that higher statistical moment cannot be prescribed using the proposed formulation). This fact is consistent with the eigensystem-based characterization of material 


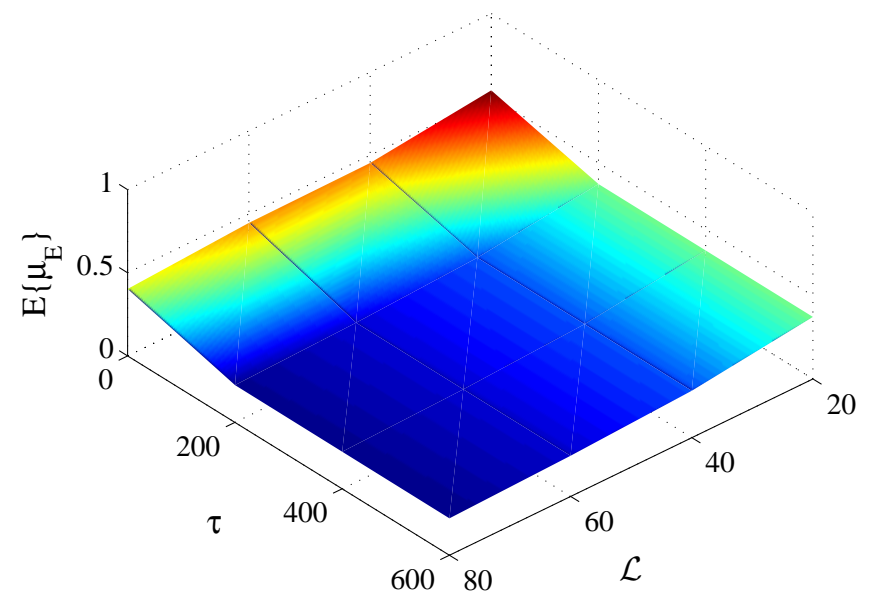

Figure 2: Plot of mapping $(\mathcal{L}, \tau) \mapsto \mathrm{E}\left\{\mu_{E}\right\}$.

symmetries, since decreasing the variances of the eigenvalues whose orders belong to $\mathcal{I}$ tends to enforce, in some sense, their relative closeness. These conclusions are also illustrated on Fig. 3, in which the plot of the p.d.f. of random variable $\mu_{E}$ for $\mathcal{L}=60, \tau=0$ (for which the result coincide with the one that would be obtained in $S E^{+}$) and $\tau=600$. Obviously, such a freedom in prescribing the mean value of stochastic anisotropy measure is intrinsically limited by the repulsion phenomena of the random eigenvalues, as well as by the choice of the symmetry class. Such results are in accordance with previous numerical results obtained for the class of transversely isotropic materials and derived without taking into account the boundedness property [18].

\section{Conclusion}

We have proposed a methodology which allows for the nonparametric probabilistic modeling of the apparent elasticity tensor associated with an elastic heterogeneous material, taking into account the classical constraints of normalization and invertibility, as well as constraints related to both boundedness and material symmetry properties. Specifically, the MaxEnt based approach unifies previous results by introducing an auxiliary positivedefinite symmetric random matrix, which is "homogeneous" to a random compliance tensor from a mechanical standpoint and for which a probabilistic model can be readily deduced by properly translating the information 


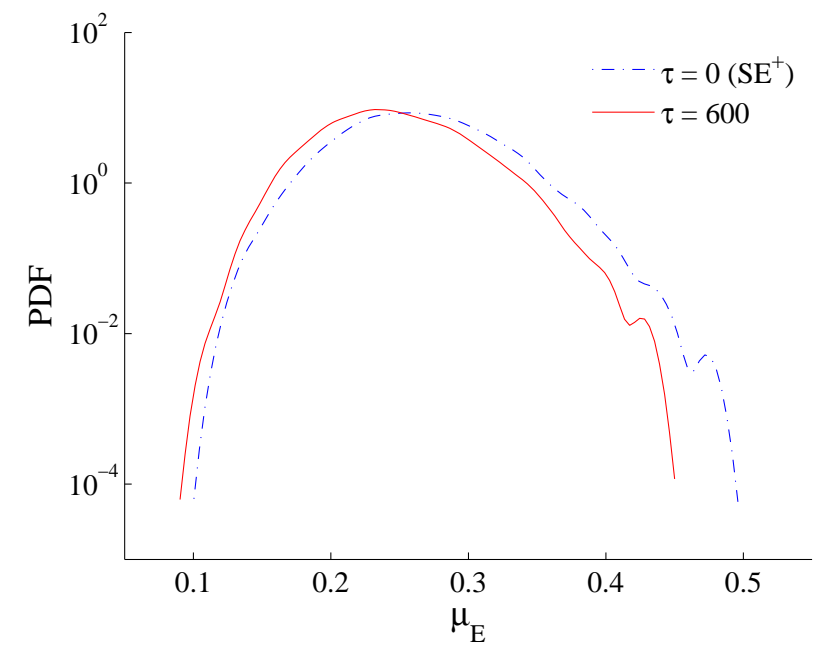

Figure 3: Plot of the p.d.f. of $\mu_{E}$ for $\mathcal{L}=60, \tau=0$ (blue dash-dot line), $\tau=600$ (red solid line).

available on the random elasticity tensor. An application to an two-phase elastic microstructure is finally provided and shows the relevance of the approach.

\section{Acknowledgments}

This work was funded by the French Research Agency (Agence Nationale de la Recherche) under TYCHE contract ANR-2010-BLAN-0904.

\section{References}

[1] V. Arsigny, P. Fillard, X. Pennec, and N. Ayache. Log-Euclidean metrics for fast and simple calculus on diffusion tensors. Magnetic Resonance in Medicine, 56:411-421, 2006.

[2] I. Babuška, F. Nobile, and R. Tempone. A stochastic collocation method for elliptic partial differential equations with random input data. SIAM Rev., 52:317-355, 2010.

[3] A. Bóna, I. Bucataru, and M.A. Slawinski. Material symmetries of elasticity tensors. The Quarterly Journal of Mechanics and Applied Mathematics, 57(4):584-598, 2004. 
[4] A. Bóna, I. Bucataru, and M.A. Slawinski. Coordinate-free characterization of the symmetry classes of elasticity tensors. Journal of Elasticity, 87:109-132, 2007.

[5] J.T. Browaeys and S. Chevrot. Decomposition of the elastic tensor and geophysical applications. Geophys. J. Int., 159:667-678, 2004.

[6] I. Bucataru and M. A. Slawinski. Invariant properties for finding distance in space of elasticity tensors. Journal of Elasticity, 94:97-114, 2009.

[7] P. Chadwick, M. Vianello, and S.C. Cowin. A new proof that the number of linear elastic symmetries is eight. Journal of the Mechanics and Physics of Solids, 49:2471-2492, 2001.

[8] D.H. Chung and W.R. Buessem. The elastic anisotropy of crystals. Journal of Applied Physics, 38(5):2010-2012, 1967.

[9] T. M. Cover and J. A. Thomas. Elements of Information Theory. John Wiley \& Sons, New-York, 2006.

[10] S.C. Cowin and M.M. Mehrabadi. On the identification of material symmetry for anisotropic elastic materials. The Quarterly Journal of Mechanics and Applied Mathematics, 40:451-476, 1987.

[11] S. Das and R. Ghanem. A bounded random matrix approach for stochastic upscaling. Multiscale Modeling ES Simulation, 8(1):296-325, 2009.

[12] F.I. Fedorov. Theory of Elastic Waves in Crystals. Plenum, New-York, 1968.

[13] S. Forte and M. Vianello. Symmetry classes for elasticity tensors. Journal of Elasticity, 43:81-108, 1996.

[14] D.C. Gazis, I. Tadjbakhsh, and R.A. Toupin. The elastic tensor of given symmetry nearest to an anisotropic elastic tensor. Acta Crystallographica, 16(9):917-922, 1963.

[15] R. G. Ghanem and P. D. Spanos. Stochastic Finite Elements: A Spectral Approach (rev. ed.). Dover Publications, 2003. 
[16] J. Guilleminot, A. Noshadravan, C. Soize, and R. G. Ghanem. A probabilistic model for bounded elasticity tensor random fields with application to polycrystalline microstructures. Computer Methods in Applied Mechanics and Engineering, to appear, 2011.

[17] J. Guilleminot and C. Soize. Non-gaussian positive-definite matrixvalued random fields with constrained eigenvalues: application to random elasticity tensors with uncertain material symmetries. Submitted for publication, 2010.

[18] J. Guilleminot and C. Soize. A stochastic model for elasticity tensors with uncertain material symmetries. International Journal of Solids and Structures, 47:3121-3130, 2010.

[19] W. K. Hastings. Monte carlo sampling methods using markov chains and their applications. Biometrika, 109:57-97, 1970.

[20] S. Hazanov and C. Huet. Order relationships for boundary condition effect in heterogeneous bodies smaller than the representative volume. Journal of the Mechanics and Physics of Solids, 42(12):1995-2011, 1994.

[21] C. Huet. Application of variational concepts to size effects in elastic heterogeneous bodies. Journal of the Mechanics and Physics of Solids, 38(6):813-841, 1990.

[22] Y.Z. Huo and G. Del Piero. On the completeness of the crystallographic symmetries in the description of the symmetries of the elasticity tensor. Journal of Elasticity, 25:203-246, 1991.

[23] E. T. Jaynes. Information theory and statistical mechanics. Physical Review, 106(4):620-630, 1957.

[24] E. T. Jaynes. Information theory and statistical mechanics. Physical Review, 108(2):171-190, 1957.

[25] T. Kanit. Notion of Representative Volume Element for Heterogeneous Materials: Statistical and Numerical Approach. PhD thesis, Ecole des Mines de Paris, 2003.

[26] T. Kanit, F. N'Guyen, S. Forest, D. Jeulin, M. Reed, and S. Singleton. Apparent and effective physical properties of heterogeneous materials: 
Representativity of samples of two materials from food industry. Computer Methods in Applied Mechanics and Engineering, 195:3960-3982, 2006.

[27] H. K. Kapur, J. N. \& Kesavan. Entropy Optimization Principles with Applications. Academic Press, San Diego, 1992.

[28] M. Kochetov and M.A. Slawinski. Estimating effective elasticity tensors from christoffel equations. Geophysics, 74(5):WB67-WB73, 2009.

[29] M. Kochetov and M.A. Slawinski. On obtaining effective orthotropic elasticity tensors. The Quarterly Journal of Mechanics and Applied Mathematics, 62(2):149-166, 2009.

[30] M. Kochetov and M.A. Slawinski. On obtaining effective transversely isotropic elasticity tensors. Journal of Elasticity, 94(1):1-13, 2009.

[31] H. Ledbetter and A. Migliori. A general elastic-anisotropy measure. Journal of Applied Physics, 100:063516-1-5, 2006.

[32] J. Löfberg. Yalmip : A toolbox for modeling and optimization in MATLAB. In Proceedings of the CACSD Conference, Taipei, Taiwan, 2004.

[33] M.M. Mehrabadi and S.C. Cowin. Eigentensors of linear anisotropic elastic materials. The Quarterly Journal of Mechanics and Applied Mathematics, 43(1):15-41, 1990.

[34] M. P. Mignolet and C. Soize. Nonparametric stochastic modeling of linear systems with prescribed variance of several natural frequencies. Probabilistic Engineering Mechanics, 23:267-278, 2008.

[35] M. Moakher. On the averaging of symmetric positivedefinite tensors. Journal of Elasticity, 82:273-296, 2006.

[36] M. Moakher and A. N. Norris. The closest elastic tensor of arbitrary symmetry to an elasticity tensor of lower symmetry. Journal of Elasticity, 85:215-263, 2006.

[37] A. N. Norris. Elastic moduli approximation of higher symmetry for the acoustical properties of an anisotropic material. Journal of the Acoutical Society of America, 119(4):2114-2121, 2006.

[38] M. Ostoja-Starzewski. Microstructural Randomness and Scaling in Mechanics of Materials. Chapman \& Hall-CRC, 2008. 
[39] S.I. Ranganathan and M. Ostoja-Starzewski. Universal elastic anisotropy index. Physical Review Letters, 101(5):055504-1-4, 2008.

[40] J. Rychlewski. On hooke's law. Prikl. Mat. Meh., 48(3):303-314, 1984.

[41] C. E. Shannon. A mathematical theory of communication. Bell System Technical Journal, 27:379-423/623-659, 1948.

[42] C. Soize. A nonparametric model of random uncertainties on reduced matrix model in structural dynamics. Probabilistic Engineering Mechanics, 15(3):277-294, 2000.

[43] C. Soize. Maximum entropy approach for modeling random uncertainties in transient elastodynamics. Journal of the Acoutical Society of America, 109(5):1979-1996, 2001.

[44] C. Soize. Non-gaussian positive-definite matrix-valued random fields for elliptic stochastic partial differential operators. Computer Methods in Applied Mechanics and Engineering, 195:26-64, 2006.

[45] C. Soize and R. G. Ghanem. Physical systems with random uncertainties: chaos representations with arbitrary probability measure. SIAM Journal on Scientific Computing, 26:395-410, 2004.

[46] Q.A. Ta, D. Clouteau, and R. Cottereau. Modeling of random anisotropic elastic media and impact on wave propagation. European Journal of Computational Mechanics, 19(1-2-3):241-253, 2010.

[47] T. C. T. Ting. Generalized cowin - mehrabadi theorems and a direct proof that the number of linear elastic symmetries is eight. International Journal of Solids and Structures, 40:7129-7142, 2003.

[48] L. Vandenberghe and S. Boyd. Semidefinite programming. SIAM Review, 38(1):49-95, 1996.

[49] X. L. Wan and G. E. Karniadakis. Multi - element generalized polynomial chaos for arbitrary probability measures. SIAM Journal on Scientific Computing, 28(3):901-928, 2006.

[50] N. Wiener. The homogeneous chaos. American Journal of Mathematics, 60:897-936, 1938 .

[51] C. Zener. Elasticity and Anelasticity of Metals. The University of Chicago Press, Chicago, 1948. 